\title{
Constant-Time Foundations for the New Spectre Era
}

\author{
Sunjay Cauligi \\ Craig Disselkoen \\ Klaus v. Gleissenthall \\ UC San Diego, USA
}

\author{
Tamara Rezk \\ INRIA Sophia Antipolis, \\ France
}

\author{
Gilles Barthe \\ MPI for Security and \\ Privacy, Germany \\ IMDEA Software Institute, \\ Spain
}

\begin{abstract}
The constant-time discipline is a software-based countermeasure used for protecting high assurance cryptographic implementations against timing side-channel attacks. Constanttime is effective (it protects against many known attacks), rigorous (it can be formalized using program semantics), and amenable to automated verification. Yet, the advent of microarchitectural attacks makes constant-time as it exists today far less useful.

This paper lays foundations for constant-time programming in the presence of speculative and out-of-order execution. We present an operational semantics and a formal definition of constant-time programs in this extended setting. Our semantics eschews formalization of microarchitectural features (that are instead assumed under adversary control), and yields a notion of constant-time that retains the elegance and tractability of the usual notion. We demonstrate the relevance of our semantics in two ways: First, by contrasting existing Spectre-like attacks with our definition of constant-time. Second, by implementing a static analysis tool, Pitchfork, which detects violations of our extended constant-time property in real world cryptographic libraries.
\end{abstract}

CCS Concepts: - Security and privacy $\rightarrow$ Formal security models; Side-channel analysis and countermeasures.

Keywords: Spectre; speculative execution; semantics; static analysis

\section{ACM Reference Format:}

Sunjay Cauligi, Craig Disselkoen, Klaus v. Gleissenthall, Dean Tullsen, Deian Stefan, Tamara Rezk, and Gilles Barthe. 2020. ConstantTime Foundations for the New Spectre Era. In Proceedings of the 41st ACM SIGPLAN International Conference on Programming Language Design and Implementation (PLDI '20), June 15-20, 2020, London, UK. ACM, New York, NY, USA, 14 pages. https://doi.org/10.1145/ 3385412.3385970

Permission to make digital or hard copies of part or all of this work for personal or classroom use is granted without fee provided that copies are not made or distributed for profit or commercial advantage and that copies bear this notice and the full citation on the first page. Copyrights for thirdparty components of this work must be honored. For all other uses, contact the owner/author(s).

PLDI '20, fune 15-20, 2020, London, UK

(C) 2020 Copyright held by the owner/author(s).

ACM ISBN 978-1-4503-7613-6/20/06.

https://doi.org/10.1145/3385412.3385970

\section{Introduction}

Protecting secrets in software is hard. Security and cryptography engineers must write programs that protect secrets, both at the source level and when they execute on real hardware. Unfortunately, hardware too easily divulges information about a program's execution via timing side-channelse.g., an attacker can learn secrets by simply observing (via timing) the effects of a program on the hardware cache [16].

The most robust way to deal with timing side-channels is via constant-time programming-the paradigm used to implement almost all modern cryptography [2, 12, 13, 26, 27]. Constant-time programs can neither branch on secrets nor access memory based on secret data. ${ }^{1}$ These restrictions ensure that programs do not leak secrets via timing sidechannels on hardware without microarchitectural features.

Unfortunately, these guarantees are moot for most modern hardware: Spectre [20], Meltdown [22], ZombieLoad [29], RIDL [32], and Fallout [5] are all dramatic examples of attacks that exploit microarchitectural features. These attacks reveal that code that is deemed constant-time in the usual sense may, in fact, leak information on processors with microarchitectural features. The decade-old constant-time recipes are no longer enough. ${ }^{2}$

In this work, we lay the foundations for constant-time in the presence of microarchitectural features that have been exploited in recent attacks: out-of-order and speculative execution. We focus on constant-time for two key reasons. First, impact: constant-time programming is largely used in real-world crypto libraries-and high-assurance code-where developers already go to great lengths to eliminate leaks via side-channels. Second, foundations: constant-time programming is already rooted in foundations, with well-defined semantics $[4,9]$. These semantics consider very powerful attackers-e.g., attackers in [4] have control over the cache and the scheduler. An advantage of considering powerful attackers is that the semantics can overlook many hardware details-e.g., since the cache is adversarially controlled, there is no point in modeling it precisely-making constant-time amenable to automated verification and enforcement.

Contributions. We first define a semantics for an abstract, three-stage (fetch, execute, and retire) machine. Our machine

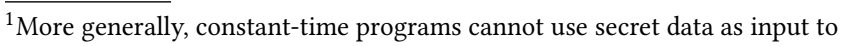
any variable-time operation-e.g., floating-point multiplication.

${ }^{2}$ OpenSSL found this situation so hopeless that they recently updated their security model to explicitly exclude "physical system side channels" [25].
} 
supports out-of-order and speculative execution by modeling reorder buffers and transient instructions, respectively. We assume that attackers have complete control over microarchitectural features (e.g., the branch target predictor) when executing a victim program and model the attacker's control over predictors using directives. This keeps our semantics simple yet powerful: our semantics abstracts over all predictors when proving security-of course, assuming that predictors themselves do not leak secrets. We further show how our semantics can be extended to capture new predictors-e.g., a hypothetical memory aliasing predictor.

We then define speculative constant-time, an extension of constant-time for machines with out-of-order and speculative execution. This definition allows us to discover microarchitectural side channels in a principled way-all four classes of Spectre attacks as classified by Canella et al. [6], for example, manifest as violations of our constant-time property.

We further use our semantics as the basis for a prototype analysis tool, Pitchfork, built on top of the angr symbolic execution engine [30]. Like other symbolic analysis tools, Pitchfork suffers from path explosion, which limits the depth of speculation we can analyze. Nevertheless, we are able to use Pitchfork to detect multiple Spectre bugs in real code. We use Pitchfork to detect leaks in the well-known Kocher test cases [19] for Spectre v1, as well as our more extensive test suite which includes Spectre v1.1 variants. More significantly, we use Pitchfork to analyze-and find leaks in-real cryptographic code from the libsodium, OpenSSL, and curve25519-donna libraries.

Open source. Pitchfork and our test suites are open source and available at https://pitchfork.programming.systems.

\section{Motivating Examples}

In this section, we show why classical constant-time programming is insufficient when attackers can exploit microarchitectural features. We do this via two example attacks and show how these attacks are captured by our semantics.

Classical constant time is not enough. Our first example consists of 3 lines of code, shown in Figure 1 (top right). The program, a variant of the classical Spectre v1 attack [20], branches on the value of register $r_{a}$ (line $\underline{1}$ ). If $r_{a}$ 's value is smaller than 4 , the program jumps to program location $\underline{2}$, where it uses $r_{a}$ to index into a public array $A$, saves the value into register $r_{b}$, and uses $r_{b}$ to index into another public array $B$. If $r_{a}$ is larger than or equal to 4 (i.e., the index is out of bounds), the program skips the two load instructions and jumps to location 4 . In a sequential execution, this program neither loads nor branches on secret values. It thus trivially satisfies the constant-time discipline.

However, modern processors do not execute sequentially. Instead, they continue fetching instructions before prior instructions are complete. In particular, a processor may continue fetching instructions beyond a conditional branch, before evaluating the branch condition. In that case, the processor guesses which branch will be taken. For example, the processor may erroneously guess that the branch condition at line 1 evaluates to true, even though $r_{a}$ contains value 9. It will therefore continue down the "true" branch speculatively. In hardware, such guesses are made by a branch prediction unit, which may have been mistrained by an adversary.

These guesses, as well as additional choices such as execution order, are directly supplied by the adversary in our semantics. We model this through a series of directives, as shown on the bottom left of Figure 1. The directive fetch: true instructs our model to speculatively follow the true branch and to place the fetched instruction at index $\overline{1}$ in the reorder buffer. Similarly, the two following fetch directives place the loads at indices $\overline{2}$ and $\overline{3}$ in the buffer. The instructions in the reorder buffer, called transient instructions, do not necessarily match the original instructions, but can contain additional information (see Table 1). For instance, the transient version of the branch instruction records which branch has been speculatively taken.

In our example, the attacker next instructs the model to execute the first load, using the directive execute $\overline{2}$. Because the bounds check has not yet been executed, the load reads from the secret element Key[1], placing the value in $r_{b}$. The attacker then issues directive execute $\overline{3}$ to execute the following load; this load's address is calculated as $44+K e y[1]$. Accessing this address affects externally visible cache state, allowing the attacker to recover $K e y[1]$ through a cache side-channel attack [16]. This is encoded by the leakage observation shown in red on the bottom right. Though this secret leakage cannot happen under sequential execution, our semantics clearly highlights the possible leak when we account for microarchitectural features.

Modeling hypothetical attacks. Next, we give an example of a hypothetical class of Spectre attack captured by our extended semantics. The attack is based on a microarchitectural feature which would allow processors to speculate whether a store and load pair might operate on the same address, and forward values between them [18, 28].

We demonstrate this attack in Figure 2. The reorder buffer, after all instructions have been fetched, is shown in the top right. The program stores the value of register $r_{b}$ into the secretKey $y_{\text {sec }}$ array and eventually loads two values from public arrays. The attacker first issues the directive execute $\overline{2}$ : value; this results in a buffer where the store instruction at $\overline{2}$ has been modified to record the resolved value $x_{\text {sec }}$. Next, the attacker issues the directive execute $\overline{7}$ : fwd $\overline{2}$, which causes the model to mispredict that the load at $\overline{7}$ aliases with the store at $\overline{2}$, and thus to forward the value $x_{\mathrm{sec}}$ to the load. The forwarded value $x_{\mathrm{sec}}$ is then used in the address $a=48+x_{\mathrm{sec}}$ of the load instruction at index $\overline{8}$. There, the loaded value $X$ is irrelevant, but the address $a$ is leaked to the attacker, allowing them to recover the secret value $x_{\mathrm{sec}}$. The speculative 


\begin{tabular}{|c|c|c|c|}
\hline \multicolumn{2}{|c|}{ Registers } & \multicolumn{2}{|r|}{ Program } \\
\hline$r$ & $\rho(r)$ & $n$ & $\mu(n)$ \\
\hline$r_{a}$ & $9_{\text {pub }}$ & $\underline{1}$ & $\operatorname{br}\left(>,\left(4, r_{a}\right), \underline{2}, \underline{4}\right)$ \\
\hline & Memory & $\underline{2}$ & $\left(r_{b}=\operatorname{load}\left(\left[40, r_{a}\right], \underline{3}\right)\right)$ \\
\hline$a$ & $\mu(a)$ & $\underline{3}$ & $\left(r_{c}=\operatorname{load}\left(\left[44, r_{b}\right], \underline{4}\right)\right)$ \\
\hline $40 . .43$ & array $A_{\text {pub }}$ & $\underline{4}$ & $\ldots$ \\
\hline $44 . .47$ & array $B_{\text {pub }}$ & & \\
\hline $48 . .4 \mathrm{~B}$ & array Key $_{\mathrm{sec}}$ & & \\
\hline
\end{tabular}

Speculative execution:

\begin{tabular}{lll} 
Directive & Effect on reorder buffer & Leakage \\
\hline fetch: true & $\overline{1} \mapsto \operatorname{br}\left(>,\left(4, r_{a}\right), \underline{2},(\underline{2}, \underline{4})\right)$ & \\
fetch & $\overline{2} \mapsto\left(r_{b}=\operatorname{load}\left(\left[40, r_{a}\right]\right)\right)$ & \\
fetch & $\overline{3} \mapsto\left(r_{c}=\operatorname{load}\left(\left[44, r_{b}\right]\right)\right)$ & \\
execute $\overline{2}$ & $\overline{2} \mapsto\left(r_{b}=\operatorname{Key}[1]_{\mathrm{sec}}\right)$ & read $49_{\text {pub }}$ \\
execute $\overline{3}$ & $\overline{3} \mapsto\left(r_{c}=X\right)$ & read $a_{\text {sec }}$ \\
& where $a=\operatorname{Key}[1]_{\mathrm{sec}}+44$ &
\end{tabular}

Figure 1. Example demonstrating a Spectre v1 attack. The branch at 1 acts as bounds check for array $A$. The execution speculatively ignores the bounds check, and leaks a byte of the secret Key.

execution continues and rolls back when the misprediction is detected (details on this are given in Section 3), but at this point, the secret has already been leaked.

As with the example in Figure 1, the program in this example follows the (sequential) constant-time discipline, yet leaks during speculative execution. But, both examples are insecure under our new notion of speculative constant-time as we discuss next.

\section{Speculative Semantics and Security}

In this section we define the notion of speculative constant time, and propose a speculative semantics that models execution on modern processors. We start by laying the groundwork for our definitions and semantics.

Configurations. A configuration $C \in$ Confs represents the state of execution at a given step. It is defined as a tuple $(\rho, \mu, n, b u f)$ where:

$\rightarrow \rho: \mathcal{R} \rightarrow \mathcal{V}$ is a map from a finite set of register names $\mathcal{R}$ to values;

$-\mu: \mathcal{V} \rightarrow \mathcal{V}$ is a memory;

- $n: \mathcal{V}$ is the current program point;

- buf $: \mathbb{N} \rightarrow$ Transinstr is the reorder buffer.

Values and labels. As a convention, we use $n$ for memory addresses that map to instructions, and $a$ for addresses that map to data. Each value is annotated with a label from a lattice of security labels with join operator $\sqcup$. For brevity, we sometimes omit public label annotation on values.

Using labels, we define an equivalence $\simeq_{\text {pub }}$ on configurations. We say that two configurations are equivalent if they coincide on public values in registers and memories.

\begin{tabular}{|c|c|c|c|}
\hline$r$ & $\begin{array}{l}\text { Registers } \\
\rho(r)\end{array}$ & $i$ & $\begin{array}{l}\text { Reorder buffer } \\
b u f(i)\end{array}$ \\
\hline$r_{a}$ & $2_{\text {pub }}$ & $\overline{2}$ & $\operatorname{store}\left(r_{b},\left[40, r_{a}\right]\right)$ \\
\hline \multirow[t]{2}{*}{$r_{b}$} & $x_{\mathrm{sec}}$ & & $\ldots$ \\
\hline & Memory & $\overline{7}$ & $\left(r_{c}=\operatorname{load}([45])\right)$ \\
\hline$a$ & $\mu(a)$ & $\overline{8}$ & $\left(r_{c}=\operatorname{load}\left(\left[48, r_{c}\right]\right)\right)$ \\
\hline $40 . .43$ & secretKey $_{\text {sec }}$ & & \\
\hline $44 . .47$ & pubArrA $A_{\text {pub }}$ & & \\
\hline $48 . .4 \mathrm{~B}$ & pubArr $B_{\text {pub }}$ & & \\
\hline
\end{tabular}

\begin{tabular}{|c|c|c|}
\hline Directive & $\begin{array}{l}\text { Speculative execution } \\
\text { Effect on buf }\end{array}$ & Leakage \\
\hline execute $\overline{2}$ : value & $\overline{2} \mapsto \operatorname{store}\left(x_{\mathrm{sec}},\left[40, r_{a}\right]\right)$ & \\
\hline execute $\overline{7}$ : fwd $\overline{2}$ & $\overline{7} \mapsto\left(r_{c}=\operatorname{load}\left([45], x_{\mathrm{sec}}, \overline{2}\right)\right)$ & \\
\hline execute $\overline{8}$ & $\overline{8} \mapsto\left(r_{c}=X\{\perp, a\}\right)$ & read $a_{\mathrm{sec}}$ \\
\hline execute $\overline{2}$ : addr & $\overline{2} \mapsto \operatorname{store}\left(r_{b}, 42_{\text {pub }}\right)$ & fwd $42_{\text {pub }}$ \\
\hline execute $\overline{7}$ & $\begin{array}{l}\{\overline{7}, \overline{8}\} \notin \text { buf } \\
\quad \text { where } a=x_{\mathrm{sec}}+48\end{array}$ & $\begin{array}{l}\text { rollback, } \\
\text { fwd } 45_{\text {pub }}\end{array}$ \\
\hline
\end{tabular}

Figure 2. Example demonstrating a hypothetical attack abusing an aliasing predictor. This attack differs from prior speculative data forwarding attacks in that branch misprediction is not needed.

Reorder buffer. The reorder buffer maps buffer indices (natural numbers) to transient instructions. We write $b u f(i)$ to denote the instruction at index $i$ in buffer $b u f$, if $i$ is in buf's domain. We write $b u f[i \mapsto \underline{\text { instr }}]$ to denote the result of extending buf with the mapping from $i$ to instr, and $b u f \backslash b u f(i)$ for the function formed by removing $i$ from $b u f$ 's domain. We write $\operatorname{buf}[j: j<i]$ to denote the restriction of buf's domain to all indices $j$, s.t. $j<i$ (i.e., removing all mappings at indices $i$ and greater). Our rules add and remove indices in a way that ensures that buf's domain will always be contiguous.

Notation. We let $\operatorname{MIN}(M)$ (resp. $\operatorname{MAX}(M))$ denote the minimum (maximum) index in the domain of a mapping $M$. We denote the empty mapping as $\emptyset$ and let $\operatorname{MIN}(\emptyset)=\operatorname{MAX}(\emptyset)=0$.

For a formula $\varphi$, we may discuss the bounded highest (lowest) index for which a formula holds. We write $\max (j)<$ $i: \varphi(j)$ to mean that $j$ is the highest index less than $i$ for which $\varphi$ holds, and define $\min (j)>i: \varphi(j)$ analogously.

Register resolve function. In Figure 3, we define the register resolve function, which we use to determine the value of a register in the presence of transient instructions in the reorder buffer. For index $i$ and register $r$, the function may (1) return the latest assignment to $r$ prior to position $i$ in the buffer, if the corresponding operation is already resolved; (2) return the value from the register map $\rho$, if there are no pending assignments to $r$ in the buffer; or (3) be undefined. Note that if the latest assignment to $r$ is yet unresolved then $\left(\right.$ buf $\left.+_{i} \rho\right)(r)=\perp$. We extend this definition to values by 
Table 1. Instructions and their transient instruction form.

Instruction Transient form(s)

\begin{tabular}{|c|c|c|c|}
\hline $\begin{array}{l}\text { arithmetic operation } \\
\text { (op specifies opcode) }\end{array}$ & $\left(r=o p\left(o p, \vec{r}, n^{\prime}\right)\right)$ & $\begin{array}{l}(r=o p(o p, \vec{r})) \\
\left(r=v_{\ell}\right)\end{array}$ & $\begin{array}{l}\text { (unresolved op) } \\
\text { (resolved value) }\end{array}$ \\
\hline conditional branch & $\operatorname{br}\left(o p, \overrightarrow{r v}, n^{\text {true }}, n^{\text {false }}\right)$ & $\begin{array}{l}\operatorname{br}\left(o p, \overrightarrow{r v}, n_{0},\left(n^{\text {true }}, n^{\text {false }}\right)\right) \\
\text { jump } n_{0}\end{array}$ & $\begin{array}{l}\text { (unresolved conditional) } \\
\text { (resolved conditional) }\end{array}$ \\
\hline $\begin{array}{r}\text { memory load } \\
(\text { at program point } n)\end{array}$ & $\left(r=\operatorname{load}\left(\vec{r}, n^{\prime}\right)\right)$ & $\begin{array}{l}(r=\operatorname{load}(\overrightarrow{r v}))^{n} \\
\left(r=\operatorname{load}\left(\overrightarrow{r v},\left(v_{\ell}, j\right)\right)\right)^{n} \\
\left(r=v_{\ell}\{\perp, a\}\right)^{n} \\
\left(r=v_{\ell}\{j, a\}\right)^{n}\end{array}$ & $\begin{array}{l}\text { (unresolved load) } \\
\text { (partially resolved load with dependency on } j \text { ) } \\
\text { (resolved load without dependencies) } \\
\text { (resolved load with dependency on } j \text { ) }\end{array}$ \\
\hline memory store & $\operatorname{store}\left(r v, \vec{r}, n^{\prime}\right)$ & $\begin{array}{l}\text { store }(r v, \vec{r}) \\
\text { store }\left(v_{\ell}, a_{\ell}\right)\end{array}$ & $\begin{array}{l}\text { (unresolved store) } \\
\text { (resolved store) }\end{array}$ \\
\hline indirect jump & $\mathrm{jmpi}(\overrightarrow{r v})$ & $\operatorname{jmpi}\left(\overrightarrow{r v}, n_{0}\right)$ & (unresolved jump predicted to $n_{0}$ ) \\
\hline function calls & $\begin{array}{l}\operatorname{call}\left(n_{f}, n_{r e t}\right) \\
\text { ret }\end{array}$ & $\begin{array}{l}\text { call } \\
\text { ret }\end{array}$ & $\begin{array}{l}\text { (unresolved call) } \\
\text { (unresolved return) }\end{array}$ \\
\hline speculation fence & fence $n$ & fence & (no resolution step) \\
\hline
\end{tabular}

$$
\left(b u f+_{i} \rho\right)(r)= \begin{cases}v_{\ell} & \text { if } \max (j)<i: \operatorname{buf}(j)=\left(r==_{-}\right) \wedge \\ & \quad \operatorname{buf}(j)=\left(r=v_{\ell}\right) \\ \rho(r) & \text { if } \forall j<i: \operatorname{buf}(j) \neq\left(r=_{-}\right) \\ \perp & \text { otherwise }\end{cases}
$$

Figure 3. Definition of the register resolve function.

defining $\left(b u f+_{i} \rho\right)\left(v_{\ell}\right)=v_{\ell}$ for all $v_{\ell} \in \mathcal{V}$, and lift it to lists of registers or values using a pointwise lifting.

\subsection{Speculative Constant-Time}

We present our new notion of constant-time security in terms of a small-step semantics, which relates program configurations, observations, and attacker directives.

Our semantics does not directly model caches, nor any of the predictors used by speculative semantics. Rather, we model externally visible effects-memory accesses and control flow-by producing a sequence of observations. We can thus reason about any possible cache implementation, as any cache eviction policy can be expressed as a function of the sequence of observations. Furthermore, exposing control flow observations directly in our semantics makes it unnecessary for us to track various other side channels. Indeed, while channels such as port contention or register renaming produce distinct measurable effects [20], they only serve to leak the path taken through the code-and thus modeling these observations separately would be redundant. For the same reason, we do not model a particular branch prediction strategy; we instead let the attacker resolve scheduling non-determinism by supplying a series of directives.
This approach has two important consequences. First, the use of observations and directives allows our semantics to remain tractable and amenable to verification. For instance, we do not need to model the behavior of the cache or any branch predictor. Second, our notion of speculative constanttime is robust, i.e., it holds for all possible branch predictors and replacement policies-assuming that they do not leak secrets directly, a condition that is achieved by all practical hardware implementations.

Given an attacker directive $d$, we use $C \underset{d}{\stackrel{o}{d}} C^{\prime}$ to denote the execution step from configuration $C$ to configuration $C^{\prime}$ that produces observation $o$. Program execution is defined from the small-step semantics in the usual style. We use $C O \|_{D}^{N} C^{\prime}$ to denote a sequence of execution steps from $C$ to $C^{\prime}$. Here $D$ and $O$ are the concatenation of the single-step directives and leakages, respectively; $N$ is the number of retired instructions, i.e., $N=\#\{d \in D \mid d=$ retire $\}$. When such a big step from $C$ to $C^{\prime}$ is possible, we say $D$ is a wellformed schedule of directives for $C$. We omit $D, N$, or $O$ when not used.

Definition 3.1 (Speculative constant-time). We say a configuration $C$ with schedule $D$ satisfies speculative constant-time $(\mathrm{SCT})$ with respect to a low-equivalence relation $\simeq_{\text {pub }}$ iff for every $C^{\prime}$ such that $C \simeq_{\text {pub }} C^{\prime}$ :

$$
C_{D} \Downarrow_{O} C_{1} \text { iff } C^{\prime}{ }_{D} \Downarrow_{O^{\prime}} C_{1}^{\prime} \text { and } C_{1} \simeq_{\text {pub }} C_{1}^{\prime} \text { and } O=O^{\prime} .
$$

A program satisfies SCT iff every initial configuration satisfies SCT under any schedule.

Aside, on sequential execution. Processors work hard to create the illusion that assembly instructions are executed sequentially. We validate our semantics by proving equivalence with respect to sequential execution. Formally, we 
define sequential schedules as schedules that execute and retire instructions immediately upon fetching them. We attach to each program a canonical sequential schedule and write $C \Downarrow_{\text {seq }}^{N} C^{\prime}$ to model execution under this canonical schedule. Our sequential validation is defined relative to an equivalence $\approx$ on configurations. Informally, two configurations are equivalent if their memories and register files are equal, even if their speculative states may be different.

Theorem 3.2 (Sequential equivalence). Let $C$ be an initial configuration and $D$ a well-formed schedule for $C$. If $C \Downarrow_{D}^{N} C_{1}$, then $C \Downarrow_{s e q}^{N} C_{2}$ and $C_{1} \approx C_{2}$.

Complete definitions, more properties, and proofs are given in [8].

\subsection{Overview of the Semantics}

As shown in Table 1, each instruction has a physical form and one or more transient forms. Our semantics operates on these instructions similar to a multi-stage processor pipeline. Physical instructions are fetched from memory and become transient instructions in the reorder buffer. They are then executed until they are fully resolved. Finally they are retired, updating the non-speculative state in the configuration.

In the rest of this section, we show how we model speculative execution (Section 3.3), memory operations (Section 3.4), aliasing prediction (Section 3.5), and fence instructions (Section 3.6). We also briefly describe indirect jumps and function calls (Section 3.7), which are presented in full in [8].

Our semantics captures a variety of existing Spectre variants, including v1 (Figure 1), v1.1 (Figure 6), and v4 (Figure 7), as well as a new hypothetical variant (Figure 2). Additional variants (e.g., v2 and ret2spec) can be expressed with the extended semantics given in [8]. Our semantics shows that these attacks violate SCT by producing observations depending on secrets.

\subsection{Speculative Execution}

We start with the semantics for conditional branches which introduce speculative execution.

Conditional branching. The physical instruction for conditional branches has the form $\operatorname{br}\left(o p, \vec{r}, n^{\text {true }}, n^{\text {false }}\right)$, where $o p$ is a Boolean operator whose result determines whether or not to execute the jump, $\vec{r}$ are the operands to op, and $n^{\text {true }}$ and $n^{\text {false }}$ are the program points for the true and false branches, respectively.

We show br's transient counterparts in Table 1. The unresolved form extends the physical instruction with a program point $n_{0}$, which is used to record the branch that is executed ( $n^{\text {true }}$ or $\left.n^{\text {false }}\right)$ speculatively, and may or may not correspond to the branch that is actually taken once op is resolved. The resolved form contains the final jump target.
Fetch. We give the rule for the fetch stage below.

$$
\begin{aligned}
& \begin{array}{l}
\text { COND-FETCH } \\
\mu(n)=\operatorname{br}\left(o p, \vec{r}, n^{\text {true }}, n^{\text {false }}\right)
\end{array} \quad i=\operatorname{MAX}(b u f)+1 \\
& b u f^{\prime}=b u f\left[i \mapsto \operatorname{br}\left(o p, \vec{r}, n^{\text {true }},\left(n^{\text {true }}, n^{\text {false }}\right)\right)\right] \\
& (\rho, \mu, n, b u f) \underset{\text { fetch: true }}{\longrightarrow}\left(\rho, \mu, n^{\text {true }}, b u f^{\prime}\right)
\end{aligned}
$$

The COND-FETCH rule speculatively executes the branch determined by a Boolean value $b$ given by the directive. We show the case for $b=$ true; the case for false is analogous. The rule updates the current program point $n$, allowing execution to continue along the specified branch. The rule then records the chosen branch $n^{\text {true }}$ (resp. $n^{\text {false }}$ ) in the transient jump instruction.

This semantics models the behavior of most modern processors. Since the target of the branch cannot be resolved in the fetch stage, speculation allows execution to continue and not stall until the branch target is resolved. In hardware, a branch predictor chooses which branch to execute; in our semantics, the directives fetch: true and fetch: false determine which of the rules to execute. This allows us to abstract over all possible predictor implementations.

Execute. Next, we describe the rules for the execute stage.

$$
\begin{aligned}
& \text { COND-EXECUTE-CORRECT } \\
& \operatorname{buf}(i)=\operatorname{br}\left(o p, \vec{r}, n_{0},\left(n^{\text {true }}, n^{\text {false }}\right)\right) \\
& \forall j<i: \operatorname{buf}(j) \neq \text { fence } \\
& \left(b u f+_{i} \rho\right)(\overrightarrow{r v})=\overrightarrow{v_{\ell}} \quad \llbracket o p\left(\overrightarrow{v_{\ell}}\right) \rrbracket=\text { true }_{\ell} \\
& \underline{n^{\text {true }}=n_{0} \quad b u f^{\prime}=b u f\left[i \mapsto \text { jump } n^{\text {true }}\right]} \\
& (\rho, \mu, n, b u f) \underset{\text { execute } i}{\stackrel{\text { jump } n_{\ell}^{\text {true }}}{\longrightarrow}}\left(\rho, \mu, n, b u f^{\prime}\right)
\end{aligned}
$$

$$
\begin{gathered}
\text { COND-EXECUTE-INCORRECT } \\
b u f(i)=\operatorname{br}\left(o p, \overrightarrow{r v}, n_{0},\left(n^{\text {true }}, n^{\text {false }}\right)\right) \\
\forall j<i: b u f(j) \neq \text { fence } \\
\frac{n^{\text {true }} \neq n_{0} \quad b u f^{\prime}=b u f[j: j<i]\left[i \mapsto \text { jump } n^{\text {true }}\right]}{(b)(\vec{r})=\overrightarrow{v_{\ell}} \quad \llbracket o p\left(\overrightarrow{v_{\ell}}\right) \rrbracket=\text { true }} \\
(\rho, \mu, n, b u f) \stackrel{\text { rollback,jump } n_{\ell}^{\text {true }}}{\stackrel{\text { execute } i}{\longrightarrow}\left(\rho, \mu, n^{\text {true }}, b u f^{\prime}\right)}
\end{gathered}
$$

Both rules evaluate the condition op via an evaluation function $\llbracket \cdot \rrbracket$. In both, the function produces true; but the false rules are analogous. The rules then compare the actual branch target $n_{\text {true }}$ against the speculatively chosen target $n_{0}$ from the fetch stage.

If the correct path was chosen during speculation, i.e., $n_{0}$ agrees with the correct branch $n^{\text {true }}$, rule COND-EXECUTECORRECT updates buf with the fully resolved jump instruction and emits an observation: jump $n_{\ell}^{\text {true }}$. This models an attacker that can observe control flow, e.g., by timing executions along different paths. The leaked observation $n^{\text {true }}$ has label $\ell$, propagated from the evaluation of the condition.

In case the wrong path was taken during speculation, i.e., the calculated branch $n^{\text {true }}$ disagrees with $n_{0}$, the semantics must roll back all execution steps along the erroneous path. For this, rule COND-EXECUTE-INCORRECT removes all entries 


\begin{tabular}{lll} 
& \multicolumn{2}{c}{ (a) Predicted correctly } \\
$i$ & Initial buf $(i)$ & buf $(i)$ after execute $\overline{4}$ \\
\hline$\overline{3}$ & $\left(r_{b}=4\right)$ & $\left(r_{b}=4\right)$ \\
$\overline{4}$ & $\operatorname{br}\left(<,\left(2, r_{a}\right), \underline{9},(\underline{9}, 12)\right)$ & jump $\underline{9}$ \\
$\overline{5}$ & $\left(r_{c}=\mathrm{op}\left(+,\left(1, r_{b}\right)\right)\right)$ & $\left(r_{c}=\mathrm{op}\left(+,\left(1, r_{b}\right)\right)\right)$
\end{tabular}

(b) Predicted incorrectly

\begin{tabular}{lll}
$i$ & Initial buf(i) & buf $(i)$ after execute $\overline{4}$ \\
\hline$\overline{3}$ & $\left(r_{b}=4\right)$ & $\left(r_{b}=4\right)$ \\
$\overline{4}$ & $\operatorname{br}\left(<,\left(2, r_{a}\right), \underline{12},(\underline{9}, \underline{12})\right)$ & jump $\underline{9}$ \\
$\overline{5}$ & $\left(r_{d}=\operatorname{op}\left(*,\left(r_{g}, r_{h}\right)\right)\right)$ & -
\end{tabular}

Figure 4. Correct and incorrect branch prediction. Initially, $r_{a}=3$. In (b), the misprediction causes a rollback to $\overline{4}$.

in buf that are newer than the current instruction (i.e., all entries $j \geq i$ ), sets the program point $n$ to the correct branch, and updates buf at index $i$ with correct value for the resolved jump instruction. Since an attacker can observer misspeculation through instruction timing [20], the rule issues a rollback observation in addition to the jump observation.

Retire. The rule for the retire stage is shown below; its only effect is to remove the jump instruction from the buffer.

$$
\begin{aligned}
& \text { JUMP-RETIRE } \\
& \frac{\operatorname{miN}(\text { buf })=i}{(i)=\text { jump } n_{0} \quad b u f^{\prime}=b u f \backslash b u f(i)} \\
& (\rho, \mu, n, b u f) \underset{\text { retire }}{\longrightarrow}\left(\rho, \mu, n, b u f^{\prime}\right)
\end{aligned}
$$

Examples. Figure 4 shows how branch prediction affects the reorder buffer. In part (a), the branch at index $\overline{4}$ is predicted correctly. The jump instruction is resolved, and execution proceeds as normal. In part (b), the branch at index $\overline{4}$ is incorrectly predicted. Upon executing the branch, the misprediction is detected, and buf is rolled back to index $\overline{4}$.

\subsection{Memory Operations}

The physical instruction for loads is $\left(r=\operatorname{load}\left(\vec{r}, n^{\prime}\right)\right)$, while the form for stores is $\operatorname{store}\left(r, \vec{r}, n^{\prime}\right)$. As before, $n^{\prime}$ is the program point of the next instruction. For loads, $r$ is the register receiving the result; for stores, $r$ is the register or value to be stored. For both loads and stores, $\overrightarrow{r v}$ is a list of operands (registers and values) which are used to calculate the operation's target address.

Transient counterparts of load and store are given in Table 1 . We annotate unresolved load instructions with the program point of the physical instruction that generated them; we omit annotations whenever not used. Unresolved and resolved store instructions share the same syntax, but for resolved stores, both address and operand are required to be single values.

Address calculation. We assume an arithmetic operator $a d d r$ which calculates target addresses for stores and loads from its operands. We leave this operation abstract in order to model a large variety of architectures. For example, in a simple addressing mode, $\llbracket a d d r(\vec{v}) \rrbracket$ might compute the sum of its operands; in an x86-style address mode, $\llbracket a d d r\left(\left[v_{1}, v_{2}, v_{3}\right]\right) \rrbracket$ might instead compute $v_{1}+v_{2} \cdot v_{3}$.

Store forwarding. Multiple transient load and store instructions may exist concurrently in the reorder buffer. In particular, there may be unresolved loads and stores that will read or write to the same address in memory. Under a naive model, we must wait to execute load instructions until all prior store instructions have been retired, in case they write to the address we will load from. Indeed, some real-world processors behave exactly this way [11].

For performance, most modern processors implement storeforwarding for memory operations: if a load reads from the same address as a prior store and the store has already been resolved, the processor can forward the resolved value to the load. The load can then proceed without waiting for the store to commit to memory [34].

To model these store forwarding semantics, we use annotations to recall if a load was resolved from memory or forwarding. A resolved load has the form $\left(r=v_{\ell}\{j, a\}\right)^{n}$, where the index $j$ records either the buffer index of the store instruction that forwarded its value to the load, or $\perp$ if the value was taken from memory. We also record the memory address $a$ associated with the data, and retain the program point $n$ of the load instruction that generated the value instruction. The resolved load otherwise behaves as a resolved value instruction (e.g., for the register resolve function).

Fetch. We now discuss the inference rules for memory operations, starting with the fetch stage.

$$
\begin{aligned}
& \begin{array}{l}
\text { SIMPLE-FETCH } \\
\mu(n) \in\{\text { op, load, store, fence }\} \quad n^{\prime}=\operatorname{next}(\mu(n)) \\
i=\operatorname{MAX}(\text { buf })+1 \quad \text { buf } f^{\prime}=b u f[i \mapsto \operatorname{transient}(\mu(n))]
\end{array} \\
& (\rho, \mu, n, b u f) \underset{\text { fetch }}{\rightleftarrows}\left(\rho, \mu, n^{\prime}, b u f^{\prime}\right)
\end{aligned}
$$

Given a fetch directive, rule SIMPLE-FETCH extends the reorder buffer buf with a new transient instruction (see Table 1). Other than load and store, the rule also applies to op and fence instructions. The transient $(\cdot)$ function simply translates the physical instruction at $\mu(n)$ to its unresolved transient form. It inserts the new, transient instruction at the first empty index in buf, and sets the current program point to the next instruction $n^{\prime}$. Note that transient $(\cdot)$ annotates the transient load instruction with its program point.

Load execution. Next, we cover the rules for the load execute stage.

$$
\begin{aligned}
& \text { LOAD-EXECUTE-NODEP } \\
& \begin{array}{r}
\text { buf }(i)=(r=\operatorname{load}(\overrightarrow{r v}))^{n} \quad \forall j<i: b u f(j) \neq \text { fence } \\
\left(b u f+_{i} \rho\right)(\overrightarrow{r v})=\overrightarrow{v_{\ell}} \quad \llbracket a d d r\left(\overrightarrow{v_{\ell}}\right) \rrbracket=a \\
\ell_{a}=\sqcup \vec{\ell} \quad \forall j<i: b u f(j) \neq \operatorname{store}(, a) \\
\mu(a)=v_{\ell} \quad b u f^{\prime}=b u f\left[i \mapsto\left(r=v_{\ell}\{\perp, a\}\right)^{n}\right] \\
(\rho, \mu, n, b u f) \underset{\text { execute } i}{\stackrel{\text { read } a_{\ell a}}{\longrightarrow}\left(\rho, \mu, n, b u f^{\prime}\right)}
\end{array}
\end{aligned}
$$




$$
\begin{aligned}
& \text { LOAD-EXECUTE-FORWARD } \\
& \begin{array}{r}
b u f(i)=(r=\operatorname{load}(\vec{v}))^{n} \quad \forall j<i: b u f(j) \neq \text { fence } \\
\left(b u f+_{i} \rho\right)(\overrightarrow{r v})=\overrightarrow{v_{\ell}} \quad \llbracket a d d r\left(\overrightarrow{v_{\ell}}\right) \rrbracket=a \quad \ell_{a}=\sqcup \vec{\ell}
\end{array} \\
& \begin{array}{r}
\max (j)<i: b u f(j)=\operatorname{store}(, a) \wedge b u f(j)=\operatorname{store}\left(v_{\ell}, a,\right) \\
b u f^{\prime}=b u f\left[i \mapsto\left(r=v_{\ell}\{j, a\}\right)^{n}\right]
\end{array} \\
& (\rho, \mu, n, b u f) \underset{\text { execute } i}{\text { fwd } a_{\ell_{a}}}\left(\rho, \mu, n, b u f^{\prime}\right)
\end{aligned}
$$

Given an execute directive for buffer index $i$, under the condition that $i$ points to an unresolved load, rule LOADEXECUTE-NODEP applies if there are no prior store instructions in buf that have a resolved, matching address. The rule first resolves the operand list $\vec{r}$ into a list of values $\overrightarrow{v_{\ell}}$, and then uses $\overrightarrow{v_{\ell}}$ to calculate the target address $a$. It then retrieves the current value $v_{\ell}$ at address $a$ from memory, and finally adds to the buffer a resolved value instruction assigning $v_{\ell}$ to the target register $r$. We annotate the value instruction with the address $a$ and $\perp$, signifying that the value comes from memory. Finally, the rule produces the observation read $a_{\ell_{a}}$, which renders the memory read at address $a$ with label $\ell_{a}$ visible to an attacker.

Rule LOAD-EXECUTE-FORWARD applies if the most recent store instruction in buf with a resolved, matching address has a resolved data value. Instead of accessing memory, the rule forwards the value from the store instruction, annotating the new value instruction with the calculated address $a$ and the index $j$ of the originating store instruction. The rule produces a fwd observation with the labeled address $a_{\ell_{a}}$. This observation captures that the attacker can determine (e.g., by observing the absence of memory access using a cache timing attack) that a forwarded value from address $a$ was found in the buffer instead of loaded from memory.

Importantly, neither of the rules has to wait for prior stores to be resolved and can proceed speculatively. This can lead to memory hazards when a more recent store to the load's address has not been resolved yet; we show how to deal with hazards in the rules for the store instruction.

Store execution. We show the rules for stores below.

$$
\begin{aligned}
& \begin{array}{c}
\text { StOre-EXecute-value } \\
b u f(i)=\operatorname{store}(r v, \overrightarrow{r v})
\end{array} \quad \forall j<i: b u f(j) \neq \text { fence } \\
& \left(b u f+_{i} \rho\right)(r v)=v_{\ell} \quad b u f^{\prime}=b u f\left[i \mapsto \operatorname{store}\left(v_{\ell}, \overrightarrow{r v}\right)\right]
\end{aligned}
$$

STORE-EXECUTE-ADDR-OK

$$
\begin{gathered}
b u f(i)=\operatorname{store}(r v, \vec{r}) \quad \forall j<i: b u f(j) \neq \text { fence } \\
\left(b u f+_{i} \rho\right)(\vec{r})=\overrightarrow{v_{\ell}} \quad \llbracket a d d r\left(\overrightarrow{v_{\ell}}\right) \rrbracket=a \quad \ell_{a}=\sqcup \vec{\ell} \\
\forall k>i: b u f(k)=\left(r=\ldots\left\{j_{k}, a_{k}\right\}\right): \\
\left(a_{k}=a \Rightarrow j_{k} \geq i\right) \wedge\left(j_{k}=i \Rightarrow a_{k}=a\right) \\
b u f^{\prime}=b u f\left[i \mapsto \operatorname{store}\left(r v, a_{\ell_{a}}\right)\right] \\
(\rho, \mu, n, b u f) \underset{\text { execute } i: \operatorname{addr}}{\text { fwd } a_{\ell_{a}}}\left(\rho, \mu, n, b u f^{\prime}\right)
\end{gathered}
$$

$$
\begin{aligned}
& \text { STORE-EXECUTE-ADDR-HAZARD } \\
& \operatorname{buf}(i)=\operatorname{store}(r, \overrightarrow{r v}) \quad \forall j<i: b u f(j) \neq \text { fence } \\
& \left(b u f+_{i} \rho\right)(\vec{r})=\overrightarrow{v_{\ell}} \quad \llbracket a d d r\left(\overrightarrow{v_{\ell}}\right) \rrbracket=a \quad \ell_{a}=\sqcup \vec{\ell} \\
& \min (k)>i: \operatorname{buf}(k)=\left(r=\ldots\left\{j_{k}, a_{k}\right\}\right)^{n_{k}}: \\
& \left(a_{k}=a \wedge j_{k}<i\right) \vee\left(j_{k}=i \wedge a_{k} \neq a\right) \\
& b u f^{\prime}=b u f[j: j<k]\left[i \mapsto \operatorname{store}\left(r v, a_{\ell_{a}}\right)\right] \\
& (\rho, \mu, n, b u f) \underset{\text { execute } i \text { addr }}{\stackrel{\text { rollback,fwd } a_{\ell_{a}}}{\longrightarrow}}\left(\rho, \mu, n_{k}, b u f^{\prime}\right)
\end{aligned}
$$

The execution of store is split into two steps: value resolution, represented by the directive execute $i$ : value, and address resolution, represented by the directive execute $i$ : addr; a schedule may have either step first. Either step may be skipped if data or address are already in immediate form.

Rule STORE-EXECUTE-ADDR-OK applies if no misprediction has been detected, i.e., if no load instruction forwarded data from an outdated store. We check this by requiring that all value instructions after the current index (indices $k>i$ ) with an address $a$ matching the current store must be using a value forwarded from a store at least as recent as this one $\left(a_{k}=a \Rightarrow j_{k} \geq i\right)$. We define $\perp<n$ for any index $n$-that is, if a future load matches the address of the current store but loaded its value from memory, we consider this a hazard.

If there is indeed a hazard, i.e., if there was a resolved load with an outdated value, the rule STORE-EXECUTE-ADDRHAZARD picks the earliest such instruction (index $k$ ) and restarts execution by resetting the instruction pointer to the program point $n_{k}$ of this instruction. It then discards all transient instructions at indices at least $k$ from the reorder buffer. As in the case of misspeculation, the rule issues a rollback observation.

Retire. Resolved loads are retired using the following rule.

$$
\begin{aligned}
& \begin{array}{l}
\text { VALUE-RETIRE } \\
\operatorname{MIN}(\text { buf })=i
\end{array} \quad \text { buf }(i)=\left(r=v_{\ell}\right) \\
& \frac{\rho^{\prime}=\rho\left[r \mapsto v_{\ell}\right] \quad \text { buf } f^{\prime}=b u f \backslash b u f(i)}{(\rho, \mu, n, b u f) \underset{\text { retire }}{\longrightarrow}\left(\rho^{\prime}, \mu, n, b u f^{\prime}\right)}
\end{aligned}
$$

This is the same retire rule used for simple value instructions (e.g., resolved op instructions). The rule updates the register map $\rho$ with the new value, and removes the instruction from the reorder buffer.

Stores are retired using the rule below.

$$
\begin{aligned}
& \text { STORE-RETIRE } \\
& \begin{array}{l}
\operatorname{MIN}(\text { buf })=i \quad \text { buf }(i)=\operatorname{store}\left(v_{\ell}, a_{\ell_{a}}\right) \\
\mu^{\prime}=\mu\left[a \mapsto v_{\ell}\right] \quad \text { buf }=b u f \backslash b u f(i)
\end{array} \\
& (\rho, \mu, n, b u f) \underset{\text { retire }}{\stackrel{\text { write } a_{\ell_{a}}}{\longrightarrow}\left(\rho, \mu^{\prime}, n, b u f^{\prime}\right)}
\end{aligned}
$$

A fully resolved store instruction retires similarly to a value instruction. However, instead of updating the register map $\rho$, rule STORE-RETIRE updates the memory $\mu$. Since an attacker can observe memory writes, the rule produces the observation write $a_{\ell_{a}}$ with the labeled address of the store.

Example. Figure 5 gives an example of store-to-load forwarding. In the starting configuration, the store at index 


\begin{tabular}{|c|c|c|}
\hline \multirow[b]{2}{*}{ starting buf } & \multicolumn{2}{|c|}{$\begin{array}{l}D=\text { execute } \overline{4} \text {; execute } \overline{3}: \text { addr } \\
\text { fwd } 43_{\text {pub }} ; \text { rollback, fwd } 43_{\text {pub }}\end{array}$} \\
\hline & buf after execute $\overline{4}$ & buf after D \\
\hline$\overline{2}$ store $\left(12,43_{\text {pub }}\right)$ & $\overline{2}$ store $\left(12,43_{\text {pub }}\right)$ & $\overline{2}$ store $\left(12,43_{\text {pub }}\right)$ \\
\hline$\overline{3}$ store $\left(20,\left[3, r_{a}\right]\right)$ & $\overline{3}$ store $\left(20,\left[3, r_{a}\right]\right)$ & $\overline{3}$ store $\left(20,43_{\text {pub }}\right)$ \\
\hline$\overline{4}\left(r_{c}=\operatorname{load}([43])\right)$ & $\overline{4}\left(r_{c}=12\{\overline{2}, 43\}\right)$ & \\
\hline
\end{tabular}

Figure 5. Store hazard caused by late execution of store addresses. The store address for $\overline{3}$ is resolved too late, causing the later load instruction to forward from the wrong store. When $\overline{3}$ 's address is resolved, the execution must be rolled back. In this example, $\llbracket a d d r(\cdot) \rrbracket$ adds its arguments.

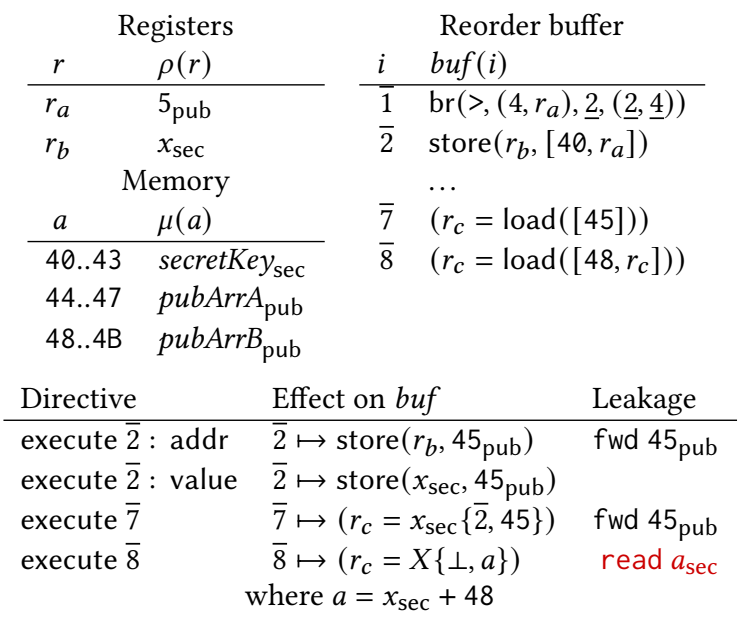

Figure 6. Example demonstrating a store-to-load Spectre v1.1 attack. A speculatively stored value is forwarded and then leaked using a subsequent load instruction.

\begin{tabular}{|c|c|c|c|}
\hline \multicolumn{2}{|c|}{ Registers } & \multicolumn{2}{|r|}{ Reorder buffer } \\
\hline$r$ & $\rho(r)$ & $i$ & buf $(i)$ \\
\hline$r_{a}$ & $40_{\text {pub }}$ & $\overline{2}$ & store $\left(0,\left[3, r_{a}\right]\right)$ \\
\hline & Memory & $\overline{3}$ & $\left(r_{c}=\operatorname{load}([43])\right)$ \\
\hline$a$ & $\mu(a)$ & $\overline{4}$ & $\left(r_{c}=\operatorname{load}\left(\left[44, r_{c}\right]\right)\right)$ \\
\hline $40 . .43$ & secretKey $_{\mathrm{sec}}$ & & \\
\hline $44 . .47$ & pubArrA $A_{\text {pub }}$ & & \\
\hline
\end{tabular}

\begin{tabular}{lll} 
Directive & Effect on buf & Leakage \\
\hline execute $\overline{3}$ & $\overline{3} \mapsto\left(r_{c}=\right.$ secretKey $\left.[3]\{\perp, 43\}\right)$ & read $43_{\text {pub }}$ \\
execute $\overline{4}$ & $\overline{4} \mapsto\left(r_{c}=X\{\perp, a\}\right)$ & read $a_{\text {sec }}$ \\
execute $\overline{2}:$ addr & $\{\overline{3}, \overline{4}\} \notin b u f$ & rollback, \\
& $\overline{2} \mapsto$ store $\left(0,43_{\mathrm{pub}}\right)$ & fwd $43_{\mathrm{pub}}$ \\
& where $a=\operatorname{secretKey}[3]_{\mathrm{sec}}+44$ &
\end{tabular}

Figure 7. Example demonstrating a v4 Spectre attack. The store is executed too late, causing later load instructions to use outdated values. $\overline{2}$ is fully resolved, while the store at index $\overline{3}$ has an unresolved address. The first directive executes the load at $\overline{4}$. This load accesses address 43 , which matches the store at index $\overline{2}$. Since this is the most recent such store and has a resolved value, the load gets the value 12 from this store. The following directive resolves the address of the store at index $\overline{3}$. This store also matches address 43 . As this store is more recent than store $\overline{2}$, this directive triggers a hazard for the load at $\overline{4}$, leading to the rollback of the load from the reorder buffer.

Capturing Spectre. We now have enough machinery to capture several variants of Spectre attacks.

We discussed how our semantics model Spectre v1 in Section 2 (Figure 1). Figure 6 shows a simple disclosure gadget using forwarding from an out-of-bounds write. In this example, a secret value $x_{\text {sec }}$ is supposed to be written to secretKey at an index $r_{a}$ as long as $r_{a}$ is within bounds. However, due to branch misprediction, the store instruction is executed despite $r_{a}$ being too large. The load instruction at index $\overline{7}$, normally benign, now aliases with the store at index $\overline{2}$, and receives the secret $x_{\text {sec }}$ instead of a public value from pubArrA. This value is then used as the address of another load instruction, causing $x_{\text {sec }}$ to leak.

Figure 7 shows a Spectre v4 vulnerability caused when a store fails to forward to a future load. In this example, the load at index $\overline{3}$ executes before the store at $\overline{2}$ calculates its address. As a result, this execution loads the outdated secret value at address 43 and leaks it, instead of using the public zeroed-out value that would be written.

\subsection{Aliasing Prediction}

We extend the memory semantics from the previous section to model aliasing prediction by introducing a new transient instruction $\left(r=\operatorname{load}\left(\vec{r},\left(v_{\ell}, j\right)\right)\right)^{n}$. This instruction represents a partially resolved load with speculatively forwarded data. As before, $r$ is the target register, $\vec{r}$ is the list of arguments for address calculation, and $n$ is the program point of the physical load instruction. The new parameters are $v_{\ell}$, the forwarded data, and $j$, the index of the originating store.

\section{Forwarding via prediction.}

$$
\begin{aligned}
& \text { LOAD-EXECUTE-FORWARDED-GUESSED } \\
& \operatorname{buf}(i)=(r=\operatorname{load}(\overrightarrow{r v}))^{n} \quad j<i \\
& \forall k<i: \operatorname{buf}(k) \neq \text { fence } \quad \operatorname{buf}(j)=\operatorname{store}\left(v_{\ell}, \vec{r}_{j}\right) \\
& \text { buf } f^{\prime}=\operatorname{buf}\left[i \mapsto\left(r=\operatorname{load}\left(\overrightarrow{r v},\left(v_{\ell}, j\right)\right)\right)^{n}\right] \\
& (\rho, \mu, n, b u f) \underset{\text { execute } i: \text { fwd } j}{\longrightarrow}\left(\rho, \mu, n, b u f^{\prime}\right)
\end{aligned}
$$

Rule LOAD-EXECUTE-FORWARDED-GUESSED implements forwarding in the presence of unresolved target addresses. Instead of forwarding the value from a store with a matching address, as in Section 3.4, the attacker can now freely choose to forward from any store with a resolved value-even if its target address is not known yet. Given a choice of which store $j$ to forward from-supplied via directive-the rule updates the reorder buffer with the new partially resolved load 
and records both the forwarded value $v_{l}$ and the buffer index $j$ of the store instruction.

Register resolve function. We extend the register resolve function $\left(b u f+_{i} \rho\right)$ to allow using values from partially resolved loads. In particular, whenever the register resolve function computes the latest resolved assignment to some register $r$, it now considers not only fully resolved value instructions, but also our new partially resolved load: whenever the latest assignment in the buffer is a partially resolved load, the register resolve function returns its value.

We now discuss the execution rules, where partially resolved loads may fully resolve against either the originating store or against memory.

\section{Resolving when originating store is in the reorder buffer.}

$$
\begin{aligned}
& \text { LOAD-EXECUTE-ADDR-OK } \\
& \operatorname{buf}(i)=\left(r=\operatorname{load}\left(\vec{r},\left(v_{\ell}, j\right)\right)\right)^{n} \\
& \left(\text { buf }+_{i} \rho\right)(\overrightarrow{r v})=\overrightarrow{v_{\ell}} \quad \llbracket a d d r\left(\overrightarrow{v_{\ell}}\right) \rrbracket=a \\
& \ell_{a}=\sqcup \vec{\ell} \quad \operatorname{buf}(j)=\operatorname{store}\left(v_{\ell}, \vec{r}_{j}\right) \wedge\left(\vec{r}_{j}=a^{\prime} \Rightarrow a^{\prime}=a\right) \\
& \forall k:(j<k<i): \operatorname{buf}(k) \neq \operatorname{store}(, a) \\
& \text { buf } f^{\prime}=\operatorname{buf}\left[i \mapsto\left(r=v_{\ell}\{j, a\}\right)^{n}\right] \\
& (\rho, \mu, n, b u f) \underset{\text { execute } i}{\stackrel{\text { fwd } a_{\ell_{a}}}{\longrightarrow}}\left(\rho, \mu, n, b u f^{\prime}\right) \\
& \text { LOAD-EXECUTE-ADDR-HAZARD } \\
& \operatorname{buf}(i)=\left(r=\operatorname{load}\left(\vec{r},\left(v_{\ell}, j\right)\right)\right)^{n^{\prime}} \\
& \left(\text { buf }+_{i} \rho\right)(\overrightarrow{r v})=\overrightarrow{v_{\ell}} \quad \llbracket a d d r\left(\overrightarrow{v_{\ell}}\right) \rrbracket=a \\
& \ell_{a}=\sqcup \vec{\ell} \quad\left(b u f(j)=\operatorname{store}\left(v_{\ell}, a^{\prime}\right) \wedge a^{\prime} \neq a\right) \vee \\
& (\exists k: j<k<i \wedge \operatorname{buf}(k)=\operatorname{store}(, a)) \\
& \text { buf } f^{\prime}=b u f[j: j<i] \\
& (\rho, \mu, n, b u f) \stackrel{\text { rollback,fwd } a_{\ell_{a}}}{\stackrel{\text { execute } i}{\hookrightarrow}}\left(\rho, \mu, n^{\prime}, b u f^{\prime}\right)
\end{aligned}
$$

To resolve $\left(r=\operatorname{load}\left(\vec{r},\left(v_{\ell}, j\right)\right)\right)^{n}$ when its originating store is still in buf, we calculate the load's actual target address $a$ and compare it against the target address of the originating store at $b u f(j)$. If the store is not followed by later stores to $a$, and either (1) the store's address is resolved and its address is indeed $a$, or (2) the store's address is still unresolved, we update the reorder buffer with an annotated value instruction (rule LOAD-EXECUTE-ADDR-OK).

If, however, either the originating store resolved to a different address (mispredicted aliasing) or a later store resolved to the same address (hazard), we roll back our execution to just before the load (rule LOAD-EXECUTE-ADDR-HAZARD).

We allow the load to execute even if the originating store has not yet resolved its address. When the store does finally resolve its address, it must check that the addresses match and that the forwarding was correct. The gray formulas in STORE-EXECUTE-ADDR-OK and STORE-EXECUTE-ADDRHAZARD (Section 3.4) perform these checks: For forwarding to be correct, all values forwarded from a store at $b u f(i)$ must have a matching annotated address $\left(\forall k>i: j_{k}=i \Rightarrow\right.$ $\left.a_{k}=a\right)$. Otherwise, if any value annotation has a mismatched address, then the instruction is rolled back $\left(j_{k}=i \wedge a_{k} \neq a\right)$.
Resolving when originating store is not in the buffer. We must also consider the case where we have delayed resolving the load address to the point where the originating store has already retired, and is no longer available in buf. If this is the case, and no other prior store instructions have a matching address, then we must check the forwarded data against memory.

$$
\begin{aligned}
& \text { LOAD-EXECUTE-ADDR-MEM-MATCH } \\
& \operatorname{buf}(i)=\left(r=\operatorname{load}\left(\overrightarrow{r v}, v_{\ell}, j\right)\right)^{n} \\
& j \notin b u f \quad\left(b u f+{ }_{i} \rho\right)(\vec{r})=\overrightarrow{v_{\ell}} \quad \ell_{a}=\sqcup \vec{\ell} \\
& \llbracket a d d r\left(\overrightarrow{v_{\ell}}\right) \rrbracket=a \quad \forall k<i: b u f(k) \neq \operatorname{store}(, a) \\
& \mu(a)=v_{\ell} \quad b u f^{\prime}=b u f\left[i \mapsto\left(r=v_{\ell}\{\perp, a\}\right)^{n}\right] \\
& (\rho, \mu, n, b u f) \underset{\text { execute } i}{\stackrel{\text { read } a_{\ell_{a}}}{\longrightarrow}}\left(\rho, \mu, n, b u f^{\prime}\right) \\
& \text { LOAD-EXECUTE-ADDR-MEM-HAZARD } \\
& \operatorname{buf}(i)=\left(r=\operatorname{load}\left(\vec{r}, v_{\ell}, j\right)\right)^{n^{\prime}} \\
& j \notin b u f \quad\left(b u f+_{i} \rho\right)(\overrightarrow{r v})=\overrightarrow{v_{\ell}} \quad \ell_{a}=\sqcup \vec{\ell} \\
& \llbracket a d d r\left(\vec{v}_{\ell}\right) \rrbracket=a \quad \forall k<i: b u f(k) \neq \operatorname{store}(, a) \\
& \underline{\mu(a)=v_{\ell^{\prime}}^{\prime} \quad v_{\ell^{\prime}}^{\prime} \neq v_{\ell} \quad b u f^{\prime}=b u f[j: j<i]} \\
& (\rho, \mu, n, b u f) \stackrel{\text { rollback,read } a_{\ell_{a}}}{\stackrel{\text { execute } i}{\hookrightarrow}}\left(\rho, \mu, n^{\prime}, b u f^{\prime}\right)
\end{aligned}
$$

If the originating store has retired, and no intervening stores match the same address, we must load the value from memory to ensure we were originally forwarded the correct value. If the value loaded from memory matches the value we were forwarded, we update the reorder buffer with a resolved load annotated as if it had been loaded from memory (rule LOAD-EXECUTE-ADDR-MEM-MATCH).

If a store different from the originating store overwrote the originally forwarded value, the value loaded from memory may not match the value we were originally forwarded. In this case we roll back execution to just before the load (rule LOAD-EXECUTE-ADDR-MEM-HAZARD).

We demonstrate these semantics in the attack shown in Figure 2. An earlier draft of this paper [7] incorrectly claimed to have a proof-of-concept exploit for this attack on real hardware.

\subsection{Speculation Barriers}

We extend our semantics with a speculation barrier instruction, fence $n$, that prevents further speculative execution until all prior instructions have been retired.

$$
\frac{\begin{array}{l}
\text { FENCE-RETIRE } \\
\operatorname{MIN}(b u f)=i \quad b u f(i)=\text { fence } \quad b u f^{\prime}=b u f \backslash b u f(i)
\end{array}}{(\rho, \mu, n, b u f) \underset{\text { retire }}{\rightleftarrows}\left(\rho, \mu, n, b u f^{\prime}\right)}
$$

The fence instruction uses SIMPLE-FETCH as its fetch rule, and its rule for retire only removes the instruction from the buffer. It does not have an execute rule. However, fence instructions affect the execution of all instructions in the reorder buffer that come after them. In prior sections, execute rules have the highlighted condition $\forall j<i: b u f(j) \neq$ fence. 


\begin{tabular}{|c|c|c|c|}
\hline$i$ & $\begin{array}{l}\text { Before executing } \overline{1} \\
\text { buf }[i]\end{array}$ & $i$ & $\begin{array}{l}\text { After } \\
\text { buf }[i]\end{array}$ \\
\hline$\overline{1}$ & $\operatorname{br}\left(>,\left(4, r_{a}\right), \underline{2},(\underline{2}, \underline{5})\right)$ & $\overline{1}$ & jump $\underline{5}$ \\
\hline$\overline{2}$ & fence & & \\
\hline$\overline{3}$ & $\left(r_{b}=\operatorname{load}\left(\left[40, r_{a}\right]\right)\right)$ & & \\
\hline$\overline{4}$ & $\left(r_{c}=\operatorname{load}\left(\left[44, r_{b}\right]\right)\right)$ & & \\
\hline
\end{tabular}

Figure 8. Example demonstrating fencing mitigation against Spectre v1 attacks. The fence instruction prevents the load instructions from executing before the br.

This condition ensures that as long as a fence instruction remains in buf, any instructions fetched after the fence cannot be executed.

We use fence instructions to restrict out-of-order execution in our semantics. Notably, we can use it to prevent attacks of the forms shown in Figures 1, 6 and 7.

Example. The example in Figure 8 shows how placing a fence instruction just after the br instruction prevents the Spectre v1 attack from Figure 1. The fence in this example prevents the load instructions at $\overline{2}$ and $\overline{3}$ from executing and forces the br to be resolved first. Evaluating the br exposes the misprediction and causes the two loads (as well as the fence) to be rolled back.

\subsection{Indirect Jumps and Return Address Prediction}

Finally, we briefly discuss two additional extensions to our semantics. First, we extend our semantics with indirect jumps. Rather than specifying jump targets directly as with the br instruction in Section 3.3, indirect jumps compute the target from a list of argument operands. The indirect jump instruction has the form jmpi $(\overrightarrow{r v})$, where $\overrightarrow{r v}$ is the list of operands for calculating the jump target. The transient form of jmpi is jmpi $\left(\vec{r}, n_{0}\right)$, where $n_{0}$ is the predicted jump target. To fetch a jmpi instruction, we use fetch: $n^{\prime}$, where $n^{\prime}$ is the speculated jump target. In all other respects, the rules for indirect jump instructions are similar to the rules for conditional branches.

Second, we extend our semantics with call and ret instructions. The call instruction has the form call $\left(n_{f}, n_{r e t}\right)$, where $n_{f}$ is the callee program point and $n_{\text {ret }}$ is the program point to return to. The return instruction is simply ret. Both the call and ret instructions have the simple transient forms call and ret. However, when fetched, they are unpacked into multiple transient instructions. Fetching a call produces the call transient instruction as well as transient instructions which will increment a stack pointer and store the return program point to memory. Fetching a ret produces a corresponding load, decrement, and jump as well as the ret transient instruction. Furthermore, the call and ret instructions respectively push and pop program points to an additional configuration state representing the return stack buffer (RSB). The RSB is used to predict the new program point upon fetching a ret.
In [8], we present detailed rules for indirect jumps, functions calls, and returns. We also show how both Spectre v2 [20] and ret2spec [23] attacks can be expressed in our semantics, as well as the retpoline mitigation [31] against Spectre v2 attacks.

\section{Detecting Violations}

We develop a tool Pitchfork based on our semantics to check for SCT violations. Pitchfork first generates a set of schedules representing various worst-case attackers. This set of schedules is far smaller than the set of all possible schedules for the program, but is nonetheless sound: if there is an SCT violation in any possible schedule, then there will be an SCT violation in one of the worst-case schedules. Pitchfork then checks for secret leakage by symbolically executing the program under each schedule.

Pitchfork only exercises a subset of our semantics; it does not detect SCT violations based on alias prediction, indirect jumps, or return stack buffers (Sections 3.5 and 3.7). Doing so would require it to generate a prohibitively large number of schedules. Nevertheless, Pitchfork still exposes attacks based on Spectre variants 1, 1.1, and 4.

We describe our schedule generation in Section 4.1, and evaluate Pitchfork on several crypto libraries in Section 4.2.

\subsection{Schedule Generation}

Given a program, Pitchfork generates a set of schedules representing various worst-case attackers. Pitchfork's schedule generation is parametrized by a speculation bound, which limits the size of the reorder buffer, and thus the depth of speculation.

In general, Pitchfork constructs worst-case schedules to maximize speculation. These schedules eagerly fetch instructions until the reorder buffer is full, i.e., the size of the reorder buffer equals the speculation bound. Once the reorder buffer is full, the schedules only retire instructions as necessary to fetch new ones.

When conditional branches are to be fetched, Pitchfork constructs schedules containing both possible outcomes: one where the branch is guessed true (fetch: true) and one where the branch is guessed false (fetch: false). For the mispredicted outcome, Pitchfork's schedules execute the branch as late as possible (i.e., it is the oldest instruction in the reorder buffer and the reorder buffer is full), which delays the rollback of mispredicted paths.

To account for the load-store forwarding hazards described in Section 3.4, Pitchfork constructs schedules containing all possible forwarding outcomes. For every load instruction $l$ in the program, Pitchfork finds all prior stores $s_{i}$ within the speculation bound that would resolve to the same address. Then, for each such store, Pitchfork constructs a schedule that would cause that store to forward its data to $l$. That is, Pitchfork constructs separate schedules [execute $s_{1}$ : 
addr; execute $l$ ], [execute $s_{2}$ : addr; execute $l$ ], and so on. Additionally, Pitchfork constructs a schedule where none of the prior stores $s_{i}$ have resolved addresses, forcing the load instruction to read from memory.

For all instructions other than conditional branches and memory operations, Pitchfork only constructs schedules where these instructions are executed eagerly and in order. Reordering of these instructions is uninteresting: either the instructions naturally commute, or data dependencies prevent the reordering (i.e., the reordered schedule is invalid for the program). This intuition matches with the property that any out-of-order execution of a given program has the same final result regardless of its schedule.

We formalize the soundness of Pitchfork's schedule construction in more detail in [8].

\subsection{Implementation and Evaluation}

We implement Pitchfork on top of the angr binary-analysis tool [30]. Pitchfork uses angr to symbolically execute a given program according to each of its worst-case schedules, flagging any resulting secret leakage.

To sanity check Pitchfork, we create and analyze a set of Spectre v1 and v1.1 test cases, and ensure we flag their SCT violations. Our test cases are based off the well-known Kocher Spectre v1 examples [19]. Since many of the Kocher examples exhibit violations even during sequential execution, we create a new set of Spectre v1 test cases which only exhibit violations when executed speculatively. We also develop a similar set of test cases for Spectre v1.1 data attacks.

Pitchfork necessarily inherits the limitations of angr's symbolic execution. For instance, angr concretizes addresses for memory operations instead of keeping them symbolic. Furthermore, exploring every speculative branch and potential store-forward within a given speculation bound leads to an explosion in state space. In our tests, we were able to support speculation bounds of up to 20 instructions. We were able to increase this bound to 250 instructions when we disabled checking for store-forwarding hazards. Though these bounds do not capture the speculation depth of some modern processors, Pitchfork still correctly finds SCT violations in all our test cases, as well as SCT violations in real-world crypto code. We consider the design and implementation of a more scalable tool future work.

4.2.1 Evaluation Procedure. To evaluate Pitchfork on real-world crypto implementations, we use the same case studies as FaCT [9], a domain-specific language and compiler for constant-time crypto code. We use FaCT's case studies for two reasons: these implementations have been verified to be (sequentially) constant-time, and their inputs have already been annotated by the FaCT authors with secrecy labels. ${ }^{3}$

We analyzed both the FaCT-generated binaries and the corresponding $\mathrm{C}$ binaries for the case studies. For each binary,

\footnotetext{
$\overline{3}$ https://github.com/PLSysSec/fact-eval
}

Table 2. A \& indicates Pitchfork found an SCT violation. A $\sim f$ indicates the violation was found only with forwarding hazard detection.

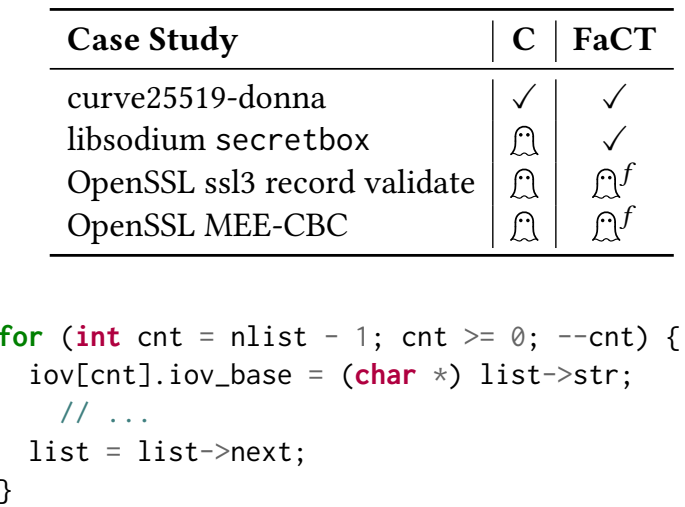

Figure 9. Vulnerable snippet from __libc__message( $).{ }^{4}$

we ran Pitchfork without forwarding hazard detection-only looking for Spectre v1 and v1.1 violations-and with a speculation bound of 250 instructions. If Pitchfork did not flag any violations, we re-enabled forwarding hazard detectionlooking for Spectre v4 violations-and ran Pitchfork with a reduced bound of 20 instructions. The reduced bound ensured that the analysis was tractable.

4.2.2 Detected Violations. Table 2 shows our results. Pitchfork did not flag any SCT violations in the curve25519-donna implementations; this is not surprising, as the curve25519donna library is a straightforward implementation of crypto primitives. Pitchfork did, however, find SCT violations (without forwarding hazard detection) in both the libsodium and OpenSSL codebases. Specifically, Pitchfork found violations in the $\mathrm{C}$ implementations of these libraries, in code ancillary to the core crypto routines. This aligns with our intuition that crypto primitives will not themselves be vulnerable to Spectre attacks, but higher-level code that interfaces with these primitives may still leak secrets. Such higher-level code is not present in the corresponding FaCT implementations, and Pitchfork did not find any violations in the FaCT code with these settings. However, with forwarding hazard detection, Pitchfork was able to find vulnerabilities even in the FaCT versions of the OpenSSL implementations. We describe two of the violations Pitchfork flagged next.

C libsodium secretbox. The libsodium codebase compiles with stack protection [15] turned on by default. This means that, for certain functions (e.g., functions with stack allocated char buffers), the compiler inserts code in the function epilogue to check if the stack was "smashed". If so, the program displays an error message and aborts. As part of printing the error message, the program calls a function __libc_message, which does printf-style string formatting. 


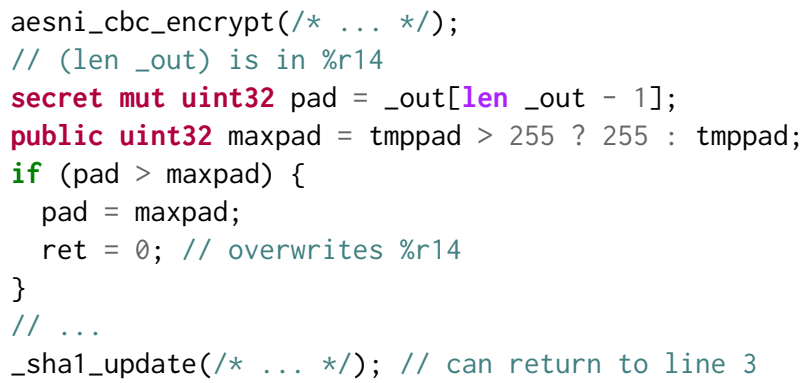

Figure 10. Vulnerable snippet from the FaCT OpenSSL MEE implementation. ${ }^{5}$

Figure 9 shows a snippet from this function which traverses a linked list. When running the $\mathrm{C}$ secretbox implementation speculatively, the processor may misspeculate on the stack tampering check and jump into the error handling code, eventually calling __libc_message. Again due to misspeculation, the processor may incorrectly proceed through the loop extra times, traversing non-existent links, eventually causing secret data to be stored into list instead of a valid address (line 4). On the following iteration of the loop, dereferencing list (line 2) causes a secret-dependent memory access.

FaCT OpenSSL MEE. In Figure 10, we show the code from the FaCT port of OpenSSL's authenticated encryption implementation. The FaCT compiler transforms the branch at lines 5-7 into straight-line constant-time code, since the variable pad is considered secret.

Initially, register \% 14 holds the length of the array _out. The processor leaks this value due to the array access on line 3; this is not a security violation, as the length is public. On line 7 , the value of $\% r 14$ is overwritten with 0 if pad > maxpad, or 1 (the initial value of ret) otherwise. Afterwards, the processor calls_sha1_update.

To return from _sha1_update, the processor must first load the return address from memory. When forwarding hazard detection is enabled, Pitchfork allows this load to speculatively receive data from stores older than the most recent store to that address (see Section 3.4). Specifically, it may receive the prior value that was stored at that location: the return address for the call to aesni_cbc_encrypt.

After the speculative return, the processor executes line 3 a second time. This time, \%r14 does not hold the public value len _out; it instead holds the value of ret, which was derived from the secret condition pad > maxpad. The processor thus accesses either_out [0] or_out[-1], leaking information about the secret value of pad via cache state.

\footnotetext{
${ }^{4}$ Code snippet taken from https://github.com/lattera/glibc/blob/ 895ef79e04a953cac1493863bcae29ad85657ee1/sysdeps/posix/libc fatal.c ${ }^{5}$ Code snippet taken from https://github.com/PLSysSec/facteval/blob/888bc6c6898a06cef54170ea273de91868ea621e/opensslmee/20170717_latest.fact
}

\section{Related Work}

Prior work on modeling speculative or out-of-order execution is concerned with correctness rather than security [1, 21]. We instead focus on security and model side-channel leakage explicitly. Moreover, we abstract away the specifics of microarchitectural features, considering them to be adversarially controlled.

Disselkoen et al. [14] explore speculation and out-of-order effects through a relaxed memory model. Their semantics sits at a higher level, and is orthogonal to our approach. They do not define a semantic notion of security that prevents Spectrelike attacks, and do not provide support for verification.

Mcilroy et al. [24] reason about micro-architectural attacks using a multi-stage pipeline semantics (though they do not define a formal security property). Their semantics models branch predictor and cache state explicitly. However, they do not model the effects of speculative barriers, nor other microarchitecture features such as store-forwarding. Thus, their semantics can only capture Spectre v1 attacks.

Both Guarnieri et al. [17] and Cheang et al. [10] define speculative semantics that are supported by tools. Their semantics handle speculation through branch predictionwhere the predictor is left abstract-but do not capture more general out-of-order execution nor other types of speculation. These works also propose new semantic notions of security (different from SCT); both essentially require that the speculative execution of a program not leak more than its sequential execution. If a program is sequentially constant-time, this additional security property is equivalent to our notion of speculative constant-time. Though our property is stronger, it is also simpler to verify: we can directly check SCT without first checking if a program is sequentially constant-time. And since we focus on cryptographic code, we directly require the stronger SCT property.

Balliu et al. [3] define a semantics in a style similar to ours. Their semantics captures various Spectre attacks, including an attack similar to our alias prediction example (Figure 2), and a new attack based on their memory ordering semantics, which our semantics cannot capture.

Finally, several tools detect Spectre vulnerabilities, but do not present semantics. The oo7 static analysis tool [33], for example, uses taint tracking to find Spectre attacks and automatically insert mitigations for several variants. $\mathrm{Wu}$ and Wang [35], on the other hand, perform cache analysis of LLVM programs under speculative execution, capturing Spectre v1 attacks.

\section{Conclusion}

We introduced a semantics for reasoning about side-channels under adversarially controlled out-of-order and speculative 
execution. Our semantics capture existing transient execution attacks-namely Spectre-but can be extended to future hardware predictors and potential attacks. We also defined a new notion of constant-time code under speculationspeculative constant-time (SCT)-and implemented a prototype tool to check if code is SCT. Our prototype, Pitchfork, discovered new vulnerabilities in real-world crypto libraries.

There are several directions for future work. Our immediate plan is to use our semantics to prove the effectiveness of existing countermeasures (e.g., retpolines) and to justify new countermeasures.

\section{Acknowledgments}

We thank the anonymous PLDI and PLDI AEC reviewers and our shepherd James Bornholt for their suggestions and insightful comments. We thank David Kaplan from AMD for his detailed analysis of our proof-of-concept exploit that we incorrectly thought to be abusing an aliasing predictor. We also thank Natalie Popescu for her aid in editing and formatting this paper. This work was supported in part by gifts from Cisco and Fastly, by the NSF under Grant Number CCF-1918573, by ONR Grant N000141512750, and by the CONIX Research Center, one of six centers in JUMP, a Semiconductor Research Corporation (SRC) program sponsored by DARPA.

\section{References}

[1] Jade Alglave, Anthony Fox, Samin Ishtiaq, Magnus O. Myreen, Susmit Sarkar, Peter Sewell, and Francesco Zappa Nardelli. 2009. The Semantics of Power and ARM Multiprocessor Machine Code. In Proceedings of the 4th Workshop on Declarative Aspects of Multicore Programming.

[2] Arm Mbed. [n.d.]. mbed TLS. Retrieved May 16, 2018 from https: //github.com/armmbed/mbedtls

[3] Musard Balliu, Mads Dam, and Roberto Guanciale. 2019. InSpectre: Breaking and Fixing Microarchitectural Vulnerabilities by Formal Analysis. arXiv:1911.00868 [cs.CR]

[4] Gilles Barthe, Gustavo Betarte, Juan Campo, Carlos Luna, and David Pichardie. 2014. System-level non-interference for constant-time cryptography. In Proceedings of the 2014 ACM SIGSAC Conference on Computer and Communications Security. ACM.

[5] Claudio Canella, Daniel Genkin, Lukas Giner, Daniel Gruss, Moritz Lipp, Marina Minkin, Daniel Moghimi, Frank Piessens, Michael Schwarz, Berk Sunar, Jo Van Bulck, and Yuval Yarom. 2019. Fallout: Leaking Data on Meltdown-resistant CPUs. In Proceedings of the 2019 ACM SIGSAC Conference on Computer and Communications Security. ACM.

[6] Claudio Canella, Jo Van Bulck, Michael Schwarz, Moritz Lipp, Benjamin von Berg, Philipp Ortner, Frank Piessens, Dmitry Evtyushkin, and Daniel Gruss. 2019. A Systematic Evaluation of Transient Execution Attacks and Defenses. In 28th USENIX Security Symposium. USENIX Association.

[7] Sunjay Cauligi, Craig Disselkoen, Klaus von Gleissenthall, Dean Tullsen, Deian Stefan, Tamara Rezk, and Gilles Barthe. 2019. Towards Constant-Time Foundations for the New Spectre Era. arXiv:1910.01755v2

[8] Sunjay Cauligi, Craig Disselkoen, Klaus von Gleissenthall, Dean Tullsen, Deian Stefan, Tamara Rezk, and Gilles Barthe. 2020. ConstantTime Foundations for the New Spectre Era. arXiv:1910.01755
[9] Sunjay Cauligi, Gary Soeller, Brian Johannesmeyer, Fraser Brown, Riad S. Wahby, John Renner, Benjamin Gregoire, Gilles Barthe, Ranjit Jhala, and Deian Stefan. 2019. FaCT: A DSL for timing-sensitive computation. In 40th ACM SIGPLAN Conference on Programming Language Design and Implementation. ACM.

[10] Kevin Cheang, Cameron Rasmussen, Sanjit Seshia, and Pramod Subramanyan. 2019. A Formal Approach to Secure Speculation. Cryptology ePrint Archive, Report 2019/310.

[11] Tien-Fu Chen and Jean-Loup Baer. 1992. Reducing Memory Latency via Non-blocking and Prefetching Caches. In 5th ACM International Conference on Architectural Support for Programming Languages and Operating Systems. ACM.

[12] Cryptography Coding Standard. 2016. Coding Rules. Retrieved June 9, 2017 from https://cryptocoding.net/index.php/Coding_rules

[13] Frank Denis. 2019. libsodium. Retrieved May 16, 2018 from https: //github.com/jedisct1/libsodium

[14] Craig Disselkoen, Radha Jagadeesan, Alan Jeffrey, and James Riely. 2019. The Code That Never Ran: Modeling Attacks on Speculative Evaluation. In 40th IEEE Symposium on Security and Privacy. IEEE.

[15] GCC Team. 2019. Using the GNU Compiler Collection (GCC): Instrumentation Options. Retrieved November 21, 2019 from https: //gcc.gnu.org/onlinedocs/gcc/Instrumentation-Options.html

[16] Qian Ge, Yuval Yarom, David Cock, and Gernot Heiser. 2018. A survey of microarchitectural timing attacks and countermeasures on contemporary hardware. Journal of Cryptographic Engineering (2018).

[17] Marco Guarnieri, Boris Köpf, José F. Morales, Jan Reineke, and Andrés Sánchez. 2020. SPECTECTOR: Principled Detection of Speculative Information Flows. In 41st IEEE Symposium on Security and Privacy. IEEE.

[18] Saad Islam, Ahmad Moghimi, Ida Bruhns, Moritz Krebbel, Berk Gulmezoglu, Thomas Eisenbarth, and Berk Sunar. 2019. SPOILER: Speculative Load Hazards Boost Rowhammer and Cache Attacks. In 28th USENIX Security Symposium. USENIX Association.

[19] Paul Kocher. 2018. Spectre mitigations in Microsoft's C/C++ compiler. Retrieved April 6, 2020 from https://www.paulkocher.com/doc/ MicrosoftCompilerSpectreMitigation.html

[20] Paul Kocher, Jann Horn, Anders Fogh, Daniel Genkin, Daniel Gruss, Werner Haas, Mike Hamburg, Moritz Lipp, Stefan Mangard, Thomas Prescher, Michael Schwarz, and Yuval Yarom. 2019. Spectre Attacks: Exploiting Speculative Execution. In 40th IEEE Symposium on Security and Privacy. IEEE.

[21] Shuvendu K. Lahiri, Sanjit A. Seshia, and Randal E. Bryant. 2002. Modeling and Verification of Out-of-Order Microprocessors in UCLID. In International Conference on Formal Methods in Computer-Aided Design. Springer.

[22] Moritz Lipp, Michael Schwarz, Daniel Gruss, Thomas Prescher, Werner Haas, Anders Fogh, Jann Horn, Stefan Mangard, Paul Kocher, Daniel Genkin, Yuval Yarom, and Mike Hamburg. 2018. Meltdown: Reading Kernel Memory from User Space. In 27th USENIX Security Symposium. USENIX Association.

[23] Giorgi Maisuradze and Christian Rossow. 2018. ret2spec: Speculative Execution Using Return Stack Buffers. In Proceedings of the 2018 ACM SIGSAC Conference on Computer and Communications Security. ACM.

[24] Ross McIlroy, Jaroslav Sevcik, Tobias Tebbi, Ben L. Titzer, and Toon Verwaest. 2019. Spectre is here to stay: An analysis of side-channels and speculative execution. arXiv:1902.05178

[25] OpenSSL. 2019. Security Policy. Retrieved April 6, 2020 from https: //www.openssl.org/policies/secpolicy.html

[26] Thomas Pornin. 2016. Why Constant-Time Crypto? Retrieved November 15, 2018 from https://www.bearssl.org/constanttime.html

[27] Thomas Pornin. 2018. Constant-Time Toolkit. Retrieved November 15, 2018 from https://github.com/pornin/CTTK

[28] Michael Schwarz, Claudio Canella, Lukas Giner, and Daniel Gruss. 2019. Store-to-Leak Forwarding: Leaking Data on Meltdown-resistant 
CPUs. arXiv:1905.05725

[29] Michael Schwarz, Moritz Lipp, Daniel Moghimi, Jo Van Bulck, Julian Stecklina, Thomas Prescher, and Daniel Gruss. 2019. ZombieLoad: Cross-Privilege-Boundary Data Sampling. In Proceedings of the 2019 ACM SIGSAC Conference on Computer and Communications Security. ACM.

[30] Yan Shoshitaishvili, Ruoyu Wang, Christopher Salls, Nick Stephens, Mario Polino, Audrey Dutcher, John Grosen, Siji Feng, Christophe Hauser, Christopher Kruegel, and Giovanni Vigna. 2016. SoK: (State of) The Art of War: Offensive Techniques in Binary Analysis. In 37th IEEE Symposium on Security and Privacy. IEEE.

[31] Paul Turner. 2019. Retpoline: a software construct for preventing branch-target-injection. Retrieved April 6, 2020 from https://support. google.com/faqs/answer/7625886
[32] Stephan van Schaik, Alyssa Milburn, Sebastian Österlund, Pietro Frigo, Giorgi Maisuradze, Kaveh Razavi, Herbert Bos, and Cristiano Giuffrida. 2019. RIDL: Rogue In-Flight Data Load. In 40th IEEE Symposium on Security and Privacy. IEEE.

[33] Guanhua Wang, Sudipta Chattopadhyay, Ivan Gotovchits, Tulika Mitra, and Abhik Roychoudhury. 2019. oo7: Low-overhead Defense against Spectre Attacks via Program Analysis. IEEE Transactions on Software Engineering (2019).

[34] Henry Wong. 2014. Store-to-Load Forwarding and Memory Disambiguation in X86 Processors. Retrieved April 6, 2020 from https://blog.stuffedcow.net/2014/01/x86-memory-disambiguation/

[35] Meng Wu and Chao Wang. 2019. Abstract Interpretation under Speculative Execution. In 40th SIGPLAN ACM Conference on Programming Language Design and Implementation. ACM. 